\title{
Gastric pneumatosis in a small-for-gestational-age neonate
}

\author{
Luit Penninga, Markus J Werz, Jurrian C Reurings, David R Nellensteijn
}

Department of Surgery,

St Elisabeth Hospital,

Willemstad, Curaçao

Correspondence to Dr Luit Penninga, LP@ctu.dk

Accepted 12 July 2015

\section{SUMMARY}

We describe a dysmature (small-for-gestational-age) neonate born at term with multiple congenital defects, who presented with bloody diarrhoea. The abdominal $X$-ray showed gastric pneumatosis. The patient was treated conservatively with intravenous fluids and antibiotics, and recovered uneventfully. The patient underwent genetic investigation, and was diagnosed with Cornelia de Lange syndrome. Gastric pneumatosis is rare, and may be the result of neonatal sepis, gastritis, pyloric stenosis, necrotising enterocolitis of the stomach, misplacement of nasogastric tubes, or non-invasive positive pressure ventilation. Furthermore, it is speculated that gastric pneumatosis might more frequently occur with congenital, cardiac or genetic disorders.

\section{BACKGROUND}

Gastric pneumatosis is a rare condition, and may occur in neonates. Gastric pneumatosis can be caused by several conditions, for example, neonatal sepsis, gastritis, pyloric stenosis, necrotising enterocolitis of the stomach, misplacement of nasogastric tubes and non-invasive positive pressure ventilation. ${ }^{1-7}$ Appropriate diagnosis and treatment of the underlying cause of gastric pneumatosis is of utmost importance.

We report on a dysmature neonate with gastric pneumatosis. The patient was later diagnosed with Cornelia de Lange syndrome, which is characterised by microcephaly, dysmorphic features, limb anomalies and severe growth and mental retardation. Furthermore, patients with Cornelia de Lange syndrome suffer from severe gastrooesophageal reflux disease. $^{89}$

\section{CASE PRESENTATION}

A dysmature girl was born after 37 weeks and 4 days of gestation with a birth weight of $1915 \mathrm{~g}$ ( $\mathrm{P}<5 \%$ percentile), length of $41 \mathrm{~cm}(\mathrm{P}<5 \%$ percentile) and skull circumference of $30.5 \mathrm{~cm}(\mathrm{P}<5 \%$ percentile). Delivery was uneventful with APGAR scores of $10 / 10$ after 1 and $5 \mathrm{~min}$, respectively. Physical examination revealed dysmorphic characteristics and congenital defects: a left-sided clubfoot, microstomy, hypertelorism and hypoplasia of the labia majora. In the first days of life the patient showed signs of hypotonia and experienced difficulties with oral feeding, for which nasogastric feeding was started. She was admitted to the neonatal intensive care unit. One day after birth she passed normal stools, but 4 days postpartum she developed bloody diarrhoea.

\section{INVESTIGATIONS}

On physical examination, the abdomen was distended and painful. Body temperature was $37.9^{\circ} \mathrm{C}$. Biochemical investigation showed increased $\mathrm{C}$ reactive protein $(6.4 \mathrm{mg} \%)$, a reduction of thrombocytes to $36 \times 10^{9} / \mathrm{L}$ and respiratory acidosis with a $\mathrm{PH}$ of 7.2. X-ray of the abdomen showed a distended stomach and pneumatosis of the gastric wall (figure 1). After treatment, the X-ray of the abdomen was repeated and the gastric pneumatosis had disappeared. No site of infection outside the abdomen, as a possible explanation for the clinical condition of the patient, was identified. Stool cultures were negative.

\section{DIFFERENTIAL DIAGNOSIS}

Known conditions that may cause gastric pneumotosis are gastritis and gastric outlet problems, usually hypertrophic pyloric stenosis. ${ }^{10} \mathrm{~A}$ recent study showed lactobezoar to be a possible cause of gastric pneumatosis mimicking necrotising enterocolitis (NEC). ${ }^{11}$ Our patient fulfilled NEC criteria including clinical, biochemical (thrombocytopaenia) and radiological findings, apart from the usual location to have NEC. ${ }^{3}$ Furthermore, our patient might have suffered from neonatal sepsis. Although we had a high index of suspicion for NEC or neonatal sepsis, we had no pathological proof for either of

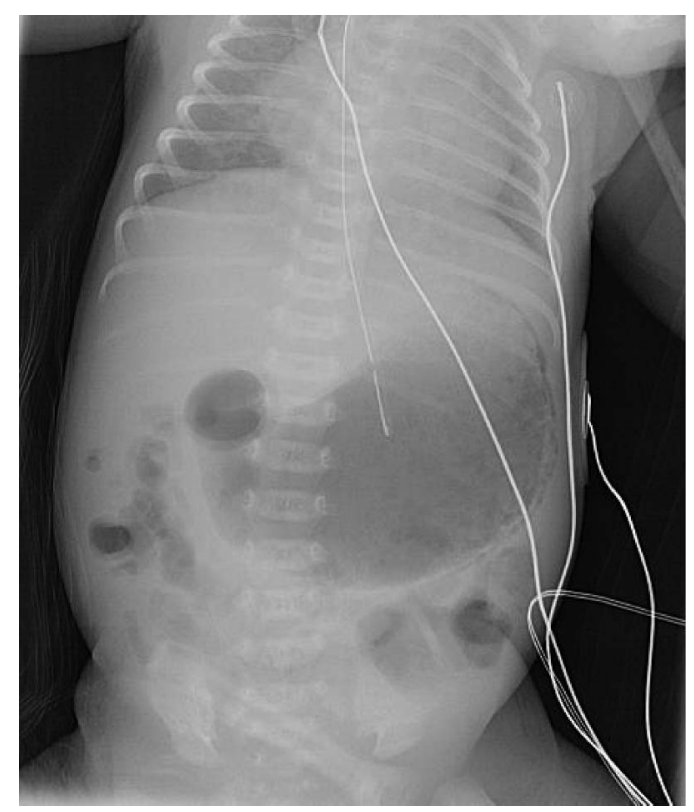

Figure 1 X-ray of the abdomen shows gastric pneumatosis. 
these conditions, and the cause of gastric pneumatosis in this patient remains speculative.

\section{TREATMENT}

The patient was treated according to the sepsis protocol, with intravenous antibiotics, amoxicillin/clavulanic acid and gentamicin, and intravenous fluids. She suffered from respiratory insufficiency for which she was intubated and continuous positive airway pressure was started.

\section{OUTCOME AND FOLLOW-UP}

The patient responded well to treatment. Two days after start of treatment the fever disappeared, and the patient passed normal stools. After 1 month she was discharged from the hospital in good condition.

At follow-up 11 months after birth, the patient was doing fine and without any abdominal symptoms. Concerning her dysmorphic appearance and congenital defects, genetic analysis was performed, and she was diagnosed with Cornelia de Lange syndrome. She is checked regularly by the paediatric cardiologist because of two small shunts in the atrial septum and a persistent ductus arteriosis.

\section{DISCUSSION}

The current case reports on a dysmature neonate with Cornelia de Lange syndrome presenting with gastric pneumatosis.

We performed an extensive literature search on gastric pneumatosis in neonates. NEC of the stomach can occur. ${ }^{1}$ NEC is the most common gastrointestinal disease in newborn infants and is characterised by necrosis of the intestinal mucosa. ${ }^{1}$ NEC occurs with an incidence of 1-3/1000 newborns, with a clear predisposition in preterm neonates. Pre-term neonates account for $90 \%$ of all NEC cases. The pathophysiology of NEC is multifactorial. The name of the disease suggests it to be solely related to the colon, though it usually affects the small bowel. ${ }^{2}$ NEC of the stomach is an extremely rare condition, and might be associated with congenital and genetic defects. $^{3-7}$

We came across multiple cases of term infants with NEC in which the condition occurred in patients with another preexisting illness such as congenital heart disease, polycythaemia, respiratory disease and neonatal sepsis. ${ }^{3-7}$ However, significant associations were never shown due to the small number of cases. ${ }^{4}$ The present case reports on a dysmature neonate with Cornelia de Lange syndrome with multiple congenital deviations including congenital heart disease. Besides dysmorphic features, mental retardation and multiple anomalies, patients with Cornelia de Lange syndrome often suffer from oesophageal

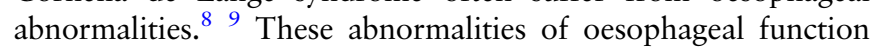
consist of GOR, swallowing incoordination and poor motility of the oesophagus, resulting in failure to thrive, and may cause aspiration pneumonia. Medical and surgical treatment for gastro-oesophageal reflux is often required in patients with Cornelia de Lange syndrome. ${ }^{8}{ }^{9}$ It remains unclear if there is any association between the occurrence of gastric pneumatosis in this patient and the Cornelia de Lange syndrome; the occurrence of gastric pneumatosis might be purely coincidental. ${ }^{8} 9$

In the literature, gastric pneumatosis is seldom related to NEC. ${ }^{1-7}{ }^{11}$ Travadi et $a l^{3}$ summarised 32-case reports of gastric pneumatosis, though only one was related with NEC. This case had a fulminant course for which a laparotomy was needed. Other conditions that cause gastric pneumatosis are gastritis and other gastric outlet obstructions, usually hypertrophic pyloric stenosis. ${ }^{3}$ Furthermore, misplacements of nasogastric tubes and non-invasive positive pressure ventilation are a possible cause of gastric pneumotosis. ${ }^{6}$ Duran $e t a l^{2}$ described two cases of gastric pneumotosis as a part of NEC. They suggested gastric pneumotosis to be a predictor of a more fulminant course of NEC, with intestinal perforation. ${ }^{2}$ Khashu described a fulminant case of NEC of the stomach in a pre-term infant for which a laparotomy was needed. ${ }^{6}$ Muller and colleagues described, similar to the present case, a non-fulminant case of NEC of the stomach after cardiogenic shock in a neonate. ${ }^{5}$

\section{Learning points}

- Pneumatosis of the stomach can be seen on conventional X-ray studies.

- Pneumatosis of the stomach can occur due to necrotising enterocolitis (NEC), neonatal sepsis, gastritis, gastric outlet obstructions (hypertrophic pyloric stenosis), misplacement of nasogastric tubes and non-invasive positive pressure ventilation.

- Although NEC predominantly affects the colon and small bowel, NEC with involvement of the stomach is a rare but possible diagnosis in neonates.

- Gastric pneumatosis might be associated with underlying predisposing physical, cardiac or congenital abnormalities.

Competing interests None declared.

Patient consent Obtained.

Provenance and peer review Not commissioned; externally peer reviewed.

\section{REFERENCES}

1 Springer A, Reck CA, Hoermann M, et al. Gastric pneumatosis in necrotizing enterocolitis. Klin Padiatr 2010;222:273.

2 Duran $R$, Vatansever U, Aksu B, et al. Gastric pneumatosis intestinalis: an indicator of intestinal perforation in preterm infants with necrotizing enterocolitis? J Pediatr Gastroenterol Nutr 2006;4:539-41.

3 Travadi JN, Patole SK, Simmer K. Gastric pneumatosis in neonates: revisited. J Paediatr Child Health 2003;39:560-2.

4 Walsh MC, Kliegman RM. Necrotizing enterocolitis: treatment based on staging criteria. Pediatr Clin North Am 1986;33:179-201.

5 Müller B, Stahr N, Knirsch W, et al. Bubbles in the heart as first sign of gastric pneumatosis. Eur J Pediatr 2014;173:1587-9.

6 Khashu M, Rudman D, Osiovich H. Gastric pneumatosis in a preterm infant. Lancet 2005;36:1808

7 Ting YJ, Chan KL, Wong SCM, et al. Gastric pneumatosis in a premature neonate. AJP Rep 2011;1:11-14.

8 Cates M, Billmire DF, Bull MJ, et al. Gatsroesophageal dysfunction in Cornelia de Lange syndrome. J Pediatr Surg 1989;34:248-51.

9 Luzzani S, Macchini F, Valadè A, et al. Gastroesophageal reflux and Cornelia de Lange syndrome; typical and atypical symptoms. Am J Med Genet 2003;119A:283-7.

10 Leonidas JC. Gastric pneumatosis in infancy. Arch Dis Child 1976;51:395-8.

11 Bos ME, Wijnen RMH, de Blaauw I. Gastric pneumatosis and rupture caused by lactobezoar. Pediatr Int 2013;55:757-60. 
Copyright 2015 BMJ Publishing Group. All rights reserved. For permission to reuse any of this content visit http://group.bmj.com/group/rights-licensing/permissions.

BMJ Case Report Fellows may re-use this article for personal use and teaching without any further permission.

Become a Fellow of BMJ Case Reports today and you can:

- Submit as many cases as you like

- Enjoy fast sympathetic peer review and rapid publication of accepted articles

- Access all the published articles

- Re-use any of the published material for personal use and teaching without further permission

For information on Institutional Fellowships contact consortiasales@bmjgroup.com

Visit casereports.bmj.com for more articles like this and to become a Fellow 